\title{
A UK survey of current ENT practice in the assessment of nasal patency
}

\section{Short title}

Assessment of nasal patency survey

\section{Type of article}

Original article

\section{Authors and affiliations}

Peter Andrews ${ }^{1}$, Jonathan Joseph ${ }^{1}$, Chia-Hung $\mathrm{Li}^{2}$, Lawrence Nip ${ }^{2}$, Thomas Jacques ${ }^{3}$, Nish Mehta ${ }^{1}$, Terence Leung ${ }^{2}$

1. Royal National Throat, Nose and Ear Hospital, London UK.

2. Department of Medical Physics and Bioengineering, University College London, London, UK

3. Department of Otolaryngology - Head and Neck Surgery, Queen's Hospital, Romford, UK.

\section{Corresponding author}

Mr Thomas Jacques

ENT Specialty Registrar

Queen's Hospital

Rom Valley Way

Romford

Essex RM7 0AG

Email: thomas.jacques@nhs.net

Telephone: 07753314101 


\begin{abstract}
Objectives

Nasal obstruction is a common presentation in ENT practice, and yet decisions on its management are challenging, with high rates of patient and clinician dissatisfaction following surgery. The aim of the study was to investigate the practice of UK ENT clinicians in the subjective and objective evaluation of nasal patency.
\end{abstract}

\title{
Design
}

Voluntary, written questionnaire.

\section{Setting}

British Academic Conference in Otolaryngology 2015, Liverpool, UK.

\section{Participants}

78 UK-based ENT professionals.

\section{Results}

78 UK based rhinologists were surveyed at the 2015 British Academic Conference in Otorhinolaryngology (BACO) from a cohort of 250 delegates attending two symposia on rhinology with a response rate of 78/250. Clinical history and examination were found to be almost universally used in the evaluation of nasal blockage. The most commonly used clinical test was the nasal misting pattern demonstration on a metal spatula (73\%), followed by the peak nasal inspiratory flow rate (19\%). The most commonly used subjective measure was the sinonasal outcome test (SNOT-22/23), with a 29\% uptake. 
$63 \%$ of the responders reported that non-availability of suitable equipment was the main reason for not using objective measures, followed by time consumption, and the lack of correlation with subjective symptom scores.

\section{Conclusions}

Our study demonstrated that British clinicians rely largely on clinical skills to evaluate nasal blockage. There is a desire for a simple, practical, non-invasive device which a) objectively measures airflow during physiological resting nasal breathing, b) correlates with subjective symptom scores, and c) is capable of simultaneous measurement of each nostril. 


\section{Introduction}

Current practice amongst ENT surgeons in nasal airway assessment in the United Kingdom (UK) is unknown, and a UK study of nasal airway assessment practice has not been performed. Nasal obstruction is a highly prevalent complaint in clinical and ENT practice ${ }^{1}$, leading to approximately $\$ 5$ billion per year in treatment costs in the USA. ${ }^{2}$ The causes and sites of obstruction are numerous; accurate diagnosis is therefore essential for effective management. There is a patient dissatisfaction rate of $30 \%$ following surgery for nasal obstruction, and hence a growing need to improve our assessment of nasal patency. ${ }^{3}$

Murrell performed a survey of USA practice in functional nasal airway assessment in $2013^{4}$ and concluded that clinical history and nasal examination with anterior rhinoscopy were the two most universal parts of a functional assessment of nasal blockage. However, it was noted that they both proved challenging to measure as an outcome owing to their subjective interpretation. Hence, the author's recommendation was to additionally perform a symptom specific questionnaire in the form of the validated Nasal Obstruction and Symptom Evaluation (NOSE) scale, nasal endoscopy to exclude a posterior obstruction (noted to be present in $28 \%$ of cases), clinical response to nasal decongestants to indicate reversible inflammatory obstruction, as well as response to Breathe Right ${ }^{\circledR}$ strips to exclude structural nasal valve obstruction.

The aim of our survey was to determine current practice in nasal patency assessment in the $\mathrm{UK}$, and to assess areas for development and determine areas of further need. 


\section{Methods}

Participants completed a written questionnaire, containing five domains:

1. Basic information: the respondent's occupation and grade, years of experience, and the number of nasal procedures recommended per week.

2. The subjective and objective methods currently utilised to carry out each of the following four tasks:

a. Diagnosing nasal obstruction,

b. Selecting patients for nasal surgery,

c. Evaluating results of nasal surgery,

d. Patient education.

3. Respondents who used at least one objective method in Part 2 were asked what they felt are the most important characteristics of these methods, and if and how they could be improved.

4. The respondents who did not report using any objective methods were asked why this was not the case.

5. The respondents were asked for the desirable attributes of a hypothetical new nasal blockage assessment device.

The setting for dissemination of the questionnaire was the British Academic Conference in Otolaryngology (BACO) 2015. Questionnaires were distributed amongst two of the BACO rhinology symposia where the lead author was presenting, with a delegate population of 250 . The questionnaires were left on the seat prior to the talks; the participants were then invited to 
complete them, for them to be collected afterwards. Although an international response was recorded, only UK responders were included.

\section{Results}

78 UK based rhinologists were surveyed at the 2015 British Academic Conference in Otorhinolaryngology (BACO), from a cohort of 250 delegates attending two symposia on rhinology, with a response rate of $31 \%$. The demographics of the 78 respondents included 40 consultant clinicians (51\%) and 24 trainees (31\%). 53\% (41) stated up to 10 years of experience in rhinology, and 47\% (37) had more than 10 years. 50\% (39) recommend up to 2 patients per week for nasal airway corrective surgery, and 33\% (26) recommend between 3 and 5 patients. Table 1 summarises this basic information.

When asked what was used in order to diagnose nasal blockage, almost all respondents used the clinical history (97\%) and physical examination (93\%). $73 \%$ also used the nasal misting pattern on a metal spatula. The most commonly-used objective measurement device was the PNIF meter, which was utilised by $19 \%$ of the respondents. The most commonly-used subjective measurement questionnaire was the SNOT-22/23, with a 29\% uptake (Figure 1).

When asked what was used in making the decision on whether to operate or not, as well as to assess the post-operative outcome, we found very similar answers were given, apart from the use of the nasal misting pattern, which decreased to $37 \% .62$ respondents commented on how they engaged in patient education: the most commonly-used method was also physical examination (65\%), followed by patient history (58\%), and 30\% used nasal spatula misting.

When asked to rate the most important characteristics of an ideal objective assessment tool; accuracy scored the highest, with $19.5 \%$ on the points-based system used, followed by ease 
of operation (16.6\% of points). Portability was the least important (3.2\%), with the remaining options assigned a similar level of importance by the respondents.

Using a Likert scale, 38 of 42 respondents (90\%) either agreed, or strongly agreed, that the existing objective methods for quantifying nasal patency could be improved. The attribute which could be improved the most was "correlation with symptom scores", with a $30 \%$ score on the points system used. The capability to "separately and simultaneously assess both nostrils" was ranked second, with $21 \%$ of the points (Figure 2).

60 participants provided their reasons for not utilising objective methods of nasal patency assessment. The most common reason was that these methods were not available to the clinician (63\%). Other common responses included the perception that objective measurement was too time-consuming (23\%), and correlated poorly with subjective symptom scores $(22 \%)$. Out of the 59 respondents who stated whether they would start to use an objective measure, if current issues were addressed, 49 respondents answered "Yes", while 10 respondents answered "No". With respect to a hypothetical new nasal blockage analyser, a clear consensus on how best to present the data to patients was not demonstrated. 


\section{Discussion}

\section{Key findings}

Our data, obtained from a range of clinicians engaged in rhinological practice, provided a new insight into the under-utilisation of objective measurements during the assessment of nasal airway patency in the UK.

Nearly all respondents used clinical history and physical examination in order to: diagnose nasal blockage, make the decision on whether to operate, and further evaluate post-operative results. Relatively few use subjective scores, with the SNOT-22/23 quality of life questionnaire being the most commonly used. Their main use amongst respondents was in the evaluation of surgical outcomes (44\%). The majority of respondents found the nasal misting pattern helpful in the diagnosis of nasal blockage, although only $37 \%$ used it as part of the assessment for surgery.

Overall, objective assessments were rarely used by the respondents in the diagnosis of nasal blockage, with only $19 \%$ using PNIF rate, which was the most commonly used test. The main reason given for not using them was lack of availability. The large majority of respondents felt that existing objective nasal patency assessment tools could be improved. The ability to assess both nostrils independently, with measurement of non-forced resting breathing, and improved correlation to symptom scores, were prioritised by respondents as desirable features of a potential novel nasal patency assessment tool.

\section{Comparison with other studies}


Murrell carried out a similar survey at the 2011 Rhinoplasty Society meeting in Boston, USA. ${ }^{4}$ The results from that survey are broadly in keeping with our data. Almost all of the respondents used anterior rhinoscopy (which in this survey was part of physical examination). Objective methods were also rarely used. 4 respondents (out of a total of 49) used rhinomanometry for preoperative assessment, which was the most frequently used objective measure. Clinical history and misting patterns were not mentioned in Murrell's study, although in the case of the former, this was more likely to be due to the reasonable assumption that it would be used universally. The main difference between the two studies was the low of patient-reported outcome measure (PROMs) usage in the UK study, compared to just under two thirds of the US respondents who did use them.

\section{Study limitations}

The interpretation of the results is potentially limited by the sample size and heterogeneity of the level of expertise amongst the respondents. There could have been a selection bias, given the trainees attending could be working with the consultants attending, and may thus have similar views. The nature of sampling (voluntary questionnaire) may have introduced a degree of selection bias; however the response rate of 74/250 was reasonable. Nevertheless, demographic information revealed a representative cohort, with similar proportions of senior and less senior respondents. Possibly due to the length of the questionnaire, not all questions were answered by all respondents. The use of a multiple-choice model for surveying opinion, rather than free text, allowed for effective and simplified data interpretation, but may have potentially added a leading element to some of the questions. 


\section{Clinical applicability of the study}

This study provided a broad overview of the current methods used by UK clinicians for the assessment of nasal obstruction, allowing other specialists to compare their practice with those of a representative cohort. UK clinicians at present base their decision-making in rhinology chiefly on clinical history and examination, with very limited use of subjective and objective measures of nasal patency.

There are a number of objective methods capable of quantifying nasal obstruction, which are most often utilised in clinical research, and occasionally employed in clinical practice. These include acoustic rhinometry, rhinomanometry and peak nasal inspiratory flow (PNIF). ${ }^{5}$

Acoustic rhinometry is user-dependent and subject to inter-user variability. It has the ability to localise the area of obstruction within the nose, through measurement of the cross-sectional area at multiple points within the nasal cavity. It is also used to assess mucosal reversibility via pre- and post-decongestant assessment. However, it does not offer a dynamic measurement of nasal airflow and hence does not directly assess function. ${ }^{6,7}$ Similarly, crosssectional imaging provides excellent anatomical definition of the nose; however, it cannot provide functional information. ${ }^{8,9}$

Rhinomanometry provides a dynamic assessment of nasal patency, and can provide unilateral assessment. It is considered to be the gold standard in objective nasal assessment; however it is considered relatively expensive, user-dependent and time-consuming. ${ }^{10}$

Peak nasal inspiratory flow rate (PNIF) is increasingly used in clinical practice and is a validated method of assessing nasal patency. However it is dependent on lung function; therefore patients with pulmonary disease may provide falsely low readings. In addition, the technique is effort-dependent and therefore subject to inter-observation variability and 
potentially user bias. It assesses forced, inspiratory nasal breathing only, which introduces non-physiological cartilaginous collapse through the Bernoulli effect. This decreases the applicability of the result to resting nasal patency, and may explain its limited correlation with subjective symptom scores. ${ }^{11}$ The technique is also unable to delineate the level of obstruction. $^{12}$

Validated patient-reported outcome measures (PROMs) are frequently used to assess the severity of nasal disease, and to determine treatment effect. Commonly-used questionnaires are the NOSE score ${ }^{12}$ and the Sinonasal Outcome Test (SNOT-22/23). The SNOT-22 score has been validated independently in patients undergoing both septorhinoplasty ${ }^{14-15}$ and endoscopic sinus surgery ${ }^{16}$.

The study confirms the desire among clinicians for a novel device capable of providing an objective, dynamic assessment of nasal airflow, which correlates well with the patient's subjective experience of blockage, and allows the patient and clinician to visualise and understand their pathology during resting nasal breathing. Direct real-time comparison of left versus right-sided airflow would be of great use in planning surgery for septoplasty or functional septorhinoplasty, and also for assessing post-operative outcomes in clinical practice and research. Equally, there is a growing need to improve patient education during their rhinological journey, and a further need to reassure a subgroup of patients, who may feel blocked subjectively, but in reality have patent nasal airways. These data have provided a knowledge base to guide the design of such a device, particularly bearing in mind the preference of clinicians for a simple test administration, best demonstrated by the popularity of the nasal misting pattern testing. We are also conducting a further study to investigate the end-user experience of patients in having their nasal blockage measured, aiming to find the optimal way of demonstrating the findings to the patient. 


\section{Conclusion}

In the current absence of a widely-adopted objective measure of nasal airflow we would recommend the use of NIPF as well as the use of the validated NOSE and SNOT 22/23 PROMs to aid patient assessment and monitor treatment outcomes. 


\section{Authorship contribution}

Peter Andrews, Jonathan Joseph and Thomas Jacques were responsible for writing the manuscript. Chia-Hung Li and Lawrence Nip designed the survey questionnaire and recruited participants. Chia-Hung Li and Terence Leung performed the analysis of results and carried out statistical tests. Terence Leung leads the design of a novel nasal patency assessment device, based in part on the results of this survey.

\section{Conflict of interest statement}

The authors declare no conflict of interest. 


\section{References}

1. Jessen M., Malm L. (1997) Definition, prevalence and development of nasal obstruction. Allergy. 52(40 Suppl), 3-6.

2. Chandra R.K., Patadia M.O., Raviv J. (2009) Diagnosis of nasal airway obstruction. Otolaryngol. Clin. North Am. 42, 207-225.

3. Lund V.J. (1989) Objective assessment of nasal obstruction. Otolaryngol. Clin. North Am. 22, 279-290.

4. Murrell G. (2013) Components of the nasal examination. Aesthet. Surg. J. 33, 38-42.

5. Hellings P.W., Scadding G., Alobid I. et al. (2012) Executive summary of European Task Force document on diagnostic tools in rhinology. Rhinology. 50, 339-52.

6. Hilberg O. (2002) Objective measurement of nasal airway dimensions using acoustic rhinometry: methodological and clinical aspects. Allergy. 57, 5-39.

7. Clement P.A., Halewyck S., Gordts F. et al. (2014) Critical evaluation of different objective techniques of nasal airway assessment: a clinical review. Eur. Arch. Otorhinolaryngol. 271, 2617-25.

8. Mamikoglu B., Houser S., Akbar I. et al. (2000) Acoustic rhinometry and computed tomography scans for the diagnosis of nasal septal deviation, with clinical correlation. Otolaryngol. Head Neck Surg. 123, $61-8$

9. Corey J.P., Gungor A., Nelson R. et al. (1997) A comparison of the nasal cross-sectional areas and volumes obtained with acoustic rhinometry and magnetic resonance imaging. Otolaryngol. Head Neck Surg. 117, 349-54.

10. Chaves C., de Andrade C.R., Ibiapina C. (2014) Objective measures for functional diagnostic of the upper airways: practical aspects. Rhinology. 52: 99-103.

11. Andrews P.J., Chowdhury N., Takhar A. et al. (2015) The need for an objective measure in septorhinoplasty surgery: are we any closer to finding an answer? Clin. Otolaryngol. 40, 698-703.

12. Nathan R.A., Eccles R., Howarth P.H. et al. (2005) Objective monitoring and nasal patency and nasal physiology in rhinitis. J. Allergy Clin. Immunol. 115, S442-59.

13. Stewart M.G., Witsell D.L., Smith T.L. et al. (2004) Development and validation of the nasal obstruction symptom evaluation (NOSE) scale. Otolaryngol. Head Neck Surg. 130, 157-163.

14. Takhar A.S., Stephens J., Randhawa P.S. et al. (2014) Validation of the sino-nasal outcome test-23 in septorhinoplasty surgery. Rhinology. 52, 320-326. 
15. Poirrier A.L., Ahluwalia S., Goodson A. et al. (2013) Is the Sino-Nasal Outcome Test-22 a suitable evaluation for septorhinoplasty? Laryngoscope. 123, 76-81.

16. Hopkins C., Gillett S., Slack R. et al. (2009) Psychometric validity of the 22-item Sinonasal Outcome Test. Clin. Otolaryngol. 34, 447-54. 


\section{Table legends}

Table 1: Basic information of survey respondents. 


\section{Figure legends}

Figure 1: Tools used by respondents in nasal airway assessment.

Figure 2: Key features of an ideal objective measure of nasal blockage.

Reference List

Poirrier AL, Ahluwalia S, Goodson A, Ellis M, Bentley M, Andrews P. Is the Sino-Nasal Outcome Test 22 a suitable evaluation for septorhinoplasty? Laryngoscope. 123[1], 76-81. 2013.

Ref Type: Journal (Full)

Hopkins C, Gillet S, Slack R, Lund V, Browne JP. Psychometric validity of the 22-item Sinonasal Outcome Test. Clin Otolaryngol. 34, 447-453. 2009.

Ref Type: Journal (Full) 